Journal Club

Editor's Note: These short, critical reviews of recent papers in the Journal, written exclusively by graduate students or postdoctoral fellows, are intended to summarize the important findings of the paper and provide additional insight and commentary. For more information on the format and purpose of the Journal Club, please see http://www.jneurosci.org/misc/ifa_features.shtml.

\title{
Cocaine Experience Guides Dynamic Changes in AMPA Receptors within the Nucleus Accumbens
}

\author{
Joshua L. Jones \\ Graduate Program in Behavioral Neuroscience, Department of Psychology, University of North Carolina, Chapel Hill, Chapel Hill, North Carolina 27599 \\ Review of Kourrich et al. (http://www.jneurosci.org/cgi/content/full/27/30/7921) and Boudreau et al. \\ (http://www.jneurosci.org/cgi/content/full/27/39/10621)
}

Increasing evidence suggests that drug addiction results from modifications of neural circuitry regulating reward learning and memory (Berke, 2003). Drugs of abuse induce long-lasting neuroadaptations at glutamatergic synapses within reward-related structures such as the nucleus accumbens (NAc) (Kauer and Malenka, 2007). Furthermore, it is well established that changes in glutamatergic synaptic transmission are mediated by alterations in expression and efficacy of AMPA receptors (Malenka, 2003). Nonetheless, a great deal regarding this neuroplasticity remains poorly understood, specifically its role in relapse to drug seeking.

There are conflicting reports on the effects of cocaine exposure and withdrawal on glutamatergic synaptic efficacy within the NAc. Previous studies have demonstrated that cocaine withdrawal results in increased spine density on medium spiny neurons (Robinson et al., 2001) as well as increased surface expression of AMPA receptors within the NAc (Boudreau and Wolf, 2005). Conversely, work has shown

Received Jan. 14, 2008; revised Feb. 6, 2008; accepted Feb. 11, 2008.

I thank Brandon J. Aragona and Robert A. Wheeler for comments during

preparation of this manuscript, and especially my advisor, Regina M. Carelli, for her invaluable mentorship.

Correspondence should be addressed to Joshua L. Jones, Graduate Program in Behavioral Neuroscience, Department of Psychology, University of North Carolina, Chapel Hill, CB\#3270 Davie Hall, Chapel Hill, NC 27599. E-mail: jljoshua@email.unc.edu.

DOI:10.1523/JNEUROSCI.0161-08.2008

Copyright $\odot$ 2008 Society for Neuroscience $\quad$ 0270-6474/08/282967-03\$15.00/0 that cocaine withdrawal is associated with reduced NAc glutamatergic signaling, through downregulation of AMPA receptor transmission (Thomas et al., 2001). Two recent articles in The Journal of Neuroscience reconcile these apparently incongruent results, by showing that differences are a function of whether animals had recent cocaine experience after cocaine withdrawal.

In the first study, Kourrich et al. (2007) used whole-cell synaptic physiology in NAc brain slices to examine changes in glutamatergic synaptic strength after repeated passive in vivo cocaine treatments. Mice were sensitized to cocaine and underwent a withdrawal period of 10-14 d, before the excitatory synaptic transmission of NAc medium spiny neurons was assessed in slice cultures. Kourrich et al. (2007)'s Figure $1 B$ (http://www.jneurosci. org/cgi/content/full/27/30/7921/F1) shows sample EPSCs from saline- and cocainetreated animals and demonstrates that glutamatergic signaling is enhanced in cocainetreated animals after cocaine withdrawal. Kourrich et al. (2007)'s Figure 1C (http:// www.jneurosci.org/cgi/content/full/27/30/ $7921 / F 1$ ) shows a significant increase in the ratio of peak evoked synaptic current in AMPA receptors (AMPARs) to that in NMDA receptors (NMDARs) after cocaine treatment and withdrawal. The authors demonstrated that this potentiation occurred through changes in AMPAR miniature EPSC frequency and amplitude [Kour- rich et al. (2007), their Fig. 2C (http:// www.jneurosci.org/cgi/content/full/27/30/ 7921/F2)], without significantly altering glutamate release probability, as measured with paired-pulse stimulation [Kourrich et al. (2007), their Fig. 2D (http://www. jneurosci.org/cgi/content/full/27/30/7921/ F2)]. Additionally, by treating the slices in $\mathrm{Mg}^{+}$-free solution in the absence and presence of the NMDAR antagonist APV, the authors showed that repeated cocaine administration did not affect synaptic NMDAR function [Kourrich et al. (2007), their Fig. 3 (http://www.jneurosci.org/cgi/ content/full/27/30/7921/F3)]. Importantly, the changes were shown to be unique to cocaine-experienced animals and a function of extended withdrawal: potentiation was absent during early withdrawal periods [Kourrich et al. (2007), their Fig. $4 B$ (http:// www.jneurosci.org/cgi/content/full/27/30/ 7921/F4)], and no change in the AMPAR/ NMDAR ratio occurred after a single injection of cocaine or saline [Kourrich et al. (2007), their Fig. 4C,D (http://www. jneurosci.org/cgi/content/full/27/30/7921/ F4)]. Interestingly, these general results are in direct contrast to previous studies, which showed the opposite effect (a decrease in AMPAR/NMDAR ratio and presence of long-term depression) (Thomas et al., 2001). However, the present experiment did not have a cocaine challenge, or reexposure to the drug, after the withdrawal period.

To replicate the previous conflicting findings, Kourrich et al. (2007) examined 
the effects of cocaine challenges on glutamatergic neurotransmission after cocaine withdrawal. They found that a single cocaine challenge injection reversed AMPAR potentiation [Kourrich et al. (2007), their Fig. 5 (http://www.jneurosci.org/ cgi/content/full/27/30/7921/F5)]. Specifically, animals that received either no challenge or saline challenge showed increased AMPAR/NMDAR ratios, whereas cocaine-challenged animals exhibited ratios that were similar to the baseline levels in naive animals. Together, this study illustrates a unique bidirectional synaptic plasticity in cocaine-experienced animals and indicates that neural responses to cocaine reexposure are highly dynamic.

Independently, Boudreau et al. (2007) quantified changes in NAc AMPAR subunits and associated kinases using protein cross-linking and immunoprecipitation studies after cocaine sensitization. Animals were trained on a cocaine or saline treatment regimen for $7 \mathrm{~d}$, after which they underwent $14 \mathrm{~d}$ of withdrawal. One group was given either a cocaine or saline challenge after withdrawal to examine the impact of cocaine reexposure on molecular subunit composition. The remaining animals were not given a challenge. After training, NAc tissue was removed and cross-linked with a membraneimpermeable protein cross-linking agent that selectively cross-links surface proteins into high-molecular weight aggregates, while leaving intracellular proteins in their normal molecular state. This process allows one to distinguish surface and intracellular proteins using SDS-PAGE and Western blotting (Boudreau and Wolf, 2005). Results from these assays demonstrated that after cocaine sensitization and withdrawal, surface levels and surface/intracellular ratios of the AMPAR subunits glutamate receptor 1 (GluR1) and GluR2 were significantly increased [Boudreau et al. (2007), their Figs. 2 (http://www.jneurosci.org/cgi/content/ full/27/39/10621/F2), 3 (http://www. jneurosci.org/cgi/content/full/27/39/ $10621 / \mathrm{F} 3)]$, which is consistent with previous findings from this group (Boudreau and Wolf, 2005). Interestingly, the GluR subunit distributions in animals given a challenge injection were markedly different: they had significantly lower surface levels and surface/intracellular ratios of GluR1 and GluR2 than unchallenged animals. These results, like those of Kourrich et al. (2007), suggest that molecular and cellular components of glutamatergic synaptic transmission shift, depending on recent cocaine experience.
Boudreau et al. (2007) also examined several trafficking molecules critically involved in AMPAR transmission. Sensitized animals exhibited an increase in extracellular signal-regulated kinase 2 (ERK2) phosphorylation in NAc after withdrawal [Boudreau et al. (2007), their Fig. 5 (http://www.jneurosci.org/cgi/ content/full/27/39/10621/F5)], but levels returned to baseline after cocaine challenge. This is one of the first demonstrations that increased ERK phosphorylation parallels increased AMPAR surface/intracellular expression ratios in striatal neurons. This is striking because ERK phosphorylation has previously been linked to AMPAR insertion during hippocampal long-term potentiation (LTP). Nonetheless, it also suggests that neuroplasticity in NAc is different from in hippocampal neurons, where LTP is not occurring during cocaine withdrawal. Together, these results suggest a unique role of MAP ( $\mathrm{mi}$ togen-activated protein) kinases, such as ERK, in AMPAR trafficking during cocaine withdrawal and subsequent reexposure.

These two studies demonstrate that bidirectional shifts in AMPAR transmission occur within a reward-related structure depending on previous cocaine experience. Both papers demonstrated that cocaine withdrawal after sensitization was associated with potentiation of glutamatergic neurotransmission, but that potentiation was abolished by cocaine pretreatment. Thus, these papers resolve incongruent findings of previous studies. Furthermore, the elaboration of this bidirectional plasticity provides a clearer understanding of how drugs alter the synaptic activity of NAc neurons, specifically during withdrawal from cocaine. The implications of these findings are critical for our future understanding of cocaine addiction and relapse.

The finding that AMPAR efficacy and surface expression in the NAc increases during withdrawal from cocaine is consistent with previous studies that suggested that increased responsiveness of excitatory synapses in the NAc plays important roles in relapse to drug-seeking behaviors. Several studies have shown that neurobiological correlates of heightened glutamate neurotransmission increase after withdrawal (Robinson et al., 2001; Boudreau and Wolf, 2005), and that increases in glutamate release within the NAc, are important for the expression of reinstatement and relapse behaviors (Kalivas et al., 2003). In contrast, electrophysiological recordings have shown that repeated co- caine exposure leads to a decrease in NAc excitability in vitro (Zhang et al., 1998), as well as NAc hypoactivity in chronic in vivo recordings (Peoples et al., 2007). It is possible that after repeated treatment with cocaine, withdrawal from the drug induces a compensatory increase in AMPAR efficacy and surface expression as a homeostatic response to manage chronic hypoactivity. Kourrich et al. (2007) and Boudreau et al. (2007) lend credence to this hypothesis by showing a clear potentiation of glutamatergic synapses, but they do not definitively answer the question.

Rapid downregulation of AMPAR neurotransmission after cocaine reexposure provides a mechanism through which behavioral responses to incentive stimuli and reward-seeking behavior can be gated through inhibition of NAc activity (Taha and Fields, 2006). It is known that drug-associated cues and contexts, stress, and drug reexposure elicit robust drug seeking and relapse after periods of withdrawal, and exposure to these incentive stimuli elicit high levels of glutamate within the NAc (Kalivas et al., 2003). We can expect that this would induce rapid AMPAR endocytosis, thus decreasing overall NAc activity. Therefore, all incentive stimuli, not just drug reexposure, should elicit this AMPAR reorganization. Two pieces of evidence suggest this is true. First, Boudreau et al. (2007) found that some cocaine-sensitized animals also exhibited conditioned locomotor sensitization in response to the saline challenge, indicating that the saline challenge served as a conditioned cue. These animals also exhibited the same decreased AMPAR subunit levels as the cocaine reexposed animals. Second, recent data presented at the Society for Neuroscience by Rothwell et al. (2007) showed that in response to an acute stress challenge in cocainesensitized animals, there was a decrease in AMPAR/NMDAR ratios, similar to the results of Kourrich et al. (2007). As such, this bidirectional AMPAR plasticity provides a potential mechanism that may mediate relapse to cocaine-seeking after reexposure to associated incentive stimuli.

Together, the studies by Kourrich et al. (2007) and Boudreau et al. (2007) provide an important characterization of NAc synaptic plasticity after cocaine withdrawal. Future lines of research should specifically address synaptic and neuronal heterogeneity of this plasticity. One possible direction is to examine differences between neurons expressing $\mathrm{D}_{1}$ and $\mathrm{D}_{2}$ dopamine receptors, because recent evidence has shown that increases in 
cocaine-induced dendritic spine density are maintained only in neurons that express $\mathrm{D}_{1}$ receptors, and not in those expressing $\mathrm{D}_{2}$ receptors (Lee et al., 2006). Additionally, as recently supposed, synapses and neurons that are differentially activated during periods of drug exposure may be more or less susceptible to druginduced neuroplasticity (Peoples et al., 2007). Further elaboration of the precise nature of experience-dependent neuroplasticity will greatly enhance our understanding of cocaine addiction and relapse.

\section{References}

Berke JD (2003) Learning and memory mechanisms involved in compulsive drug use and relapse. Methods Mol Med 79:75-101.

Boudreau AC, Wolf ME (2005) Behavioral sensitization to cocaine is associated with increased AMPA receptor surface expression in the nucleus accumbens. J Neurosci 25:9144-9151.

Boudreau AC, Reimers JM, Milovanovic M, Wolf
ME (2007) Cell surface AMPA receptors in the rat nucleus accumbens increase during cocaine withdrawal but internalize after cocaine challenge in association with altered activation of mitogen-activated protein kinases. J Neurosci 27:10621-10635.

Kalivas PW, McFarland K, Bowers S, Szumlinski K, Xi ZX, Baker D (2003) Glutamate transmission and addiction to cocaine. Ann NY Acad Sci 1003:169-175.

Kauer JA, Malenka RC (2007) Synaptic plasticity and addiction. Nat Rev Neurosci 8:844-858.

Kourrich S, Rothwell PE, Klug JR, Thomas MJ (2007) Cocaine experience controls bidirectional synaptic plasticity in the nucleus accumbens. J Neurosci 27:7921-7928.

Lee KW, Kim Y, Kim AM, Helmin K, Nairn AC, Greengard P (2006) Cocaine-induced dendritic spine formation in D1 and D2 dopamine receptor-containing medium spiny neurons in nucleus accumbens. Proc Natl Acad Sci USA 103:3399-3404.

Malenka RC (2003) Synaptic plasticity and AMPA receptor trafficking. Ann NY Acad Sci 1003:1-11.
Peoples LL, Kravitz AV, Guillem K (2007) The role of accumbal hypoactivity in cocaine addiction. ScientificWorldJournal $7: 22-45$.

Robinson TE, Gorny G, Mitton E, Kolb B (2001) Cocaine self-administration alters the morphology of dendrites and dendritic spines in the nucleus accumbens and neocortex. Synapse 39:257-266.

Rothwell PE, Kourrich S, Lacroix BL, Klug JR, Thomas MJ (2007) Shared effects of cocaine and stress on nucleus accumbens synaptic strength. Soc Neurosci Abstr 33:917.3.

Taha SA, Fields HL (2006) Inhibitions of nucleus accumbens neurons encode a gating signal for reward-directed behavior. J Neurosci 26: 217-222.

Thomas MJ, Beurrier C, Bonci A, Malenka RC (2001) Long-term depression in the nucleus accumbens: a neural correlate of behavioral sensitization to cocaine. Nat Neurosci 4:1217-1223.

Zhang XF, Hu XT, White FJ (1998) Whole-cell plasticity in cocaine withdrawal: reduced sodium currents in nucleus accumbens neurons. J Neurosci 18:488-498. 